\title{
A Rudimentary X band CW-EPR Spectrometer Based on FPGA
}

\author{
Wei Siang Eow ${ }^{a}$, Yung Szen Yapa,b,* \\ a Faculty of Science, Universiti Teknologi Malaysia, 81310 Skudai, Johor, Malaysia; \\ ${ }^{b}$ Centre for Sustainable Nanomaterials (CSNano), Universiti Teknologi Malaysia, \\ 81310 UTM Johor Bahru, Johor, Malaysia.
}

Abstract A rudimentary Electron Paramagnetic Resonance (EPR) spectrometer is designed using a field programmable gate array (FPGA) equipped with two digital-to-analog (DAC) and two analog-to-digital (ADC) channels. The single stage heterodyne setup operates at $X$ band frequencies and is used to detect EPR signals from 2,2-diphenyl-1-picrylhydrazyl (DPPH) in a loop-gap resonator. We design the loop gap resonator with 3 loops 2 gaps for high field homogeneity and moderate $\mathrm{Q}$-factor. The resonator is coupled capacitively to the coaxial cable and is designed to have an unloaded resonant frequency of $8.856 \mathrm{GHz}$ with a $\mathrm{Q}$-factor of 646.0 when critically coupled. The loaded resonant frequency is reported to be $8.668 \mathrm{GHz}$ with a Qfactor of 615.8 . Using this setup, EPR signal is successfully detected at $311.4 \mathrm{mT}$ and $8.688 \mathrm{GHz}$ with an experimental g-factor of $1.9945 \pm 0.0012$, which is very near to the standard value for DPPH.

Keywords: Electron paramagnetic resonance, resonator, EPR spectrometer

\section{Introduction}

Electron Paramagnetic Resonance (EPR) is a study of Zeeman interaction of the unpaired electron. A conventional EPR experiment is made up of 3 elements, which are external magnetic field, driven magnetic field, and an unpaired electron. External magnetic field splits the electron energy level depending on the spin quantum number while driven magnetic field provide a suitable of radio frequency (RF) magnetic field to excite the electron. EPR has a very wide field of applications such as protein study [1], biological structure study [2], defect detection [3], quantum computing [4], etc. In recent research, there was a breakthrough for large-scale spin qubit that is controlled using a global field in which the spin qubit states are manipulated by EPR [5]. The wide fields of application of EPR shows that the EPR is valuable to continue studying.

${ }^{*}$ For correspondence: yungszen@utm.my

Received: 13 Oct 2021

Accepted: 22 Nov 2021

(C) Copyright Eow and Yap. This article is distributed under the terms of the Creative Commons Attribution License, which permits unrestricted use and redistribution provided that the original author and source are credited.
In the study of EPR, there are 2 methods: continuous wave (CW) and pulsed. The continuous wave method is the method to continuously excite the electron spin using low power driven magnetic field while the pulsed method excites the spins ensemble using high power pulse with specific pulses. The continuous wave method is the simplest way to study the EPR but the applications are limited [6]. Conversely, the pulsed method more complex to study EPR but provides a way to manipulate and study the spin state of the electron [7, 8, 9]. Thus, the pulsed method is a more popular method to study the EPR. In some cases, the pulsed method still requires a continuous wave to determine the frequency of the electron spin[10].

Commercial EPR spectrometers offer multiple frequencies for continuous wave and pulsed method and some even have predesigned pulse sequence to decrease the difficulties in determining the electron spin state. As EPR is widely used in various fields of study, the versatility of the commercial product is limited as the spectrometer is designed for general uses and user friendliness. For example, the pulse shape and sequence offered by a moderate-cost commercial product has only basic sequences and 
square pulse. Square pulses contribute to significant errors in manipulating the electron spin state [11, $12,13]$. While the pulse accuracy for commercial spectrometer is enough for the majority of the fields, some fields such as quantum computing require precise control of the spin state.

For high-cost commercial pulsed EPR spectrometer, the spectrometer is built based on an arbitrary wave generator (AWG), which allows user to design their pulse shape and sequences. However, AWG is costly especially at high frequencies such as $X$ band and higher. Field programmable gate array (FPGA) is considered as the alternative to the AWG due to its flexibility, low cost, and low-profile size. However, the suitability of FPGA for signal generation and detection for EPR is still being investigated [14]. Here, an FPGA based continuous wave EPR spectrometer in $X$ band by using arbitrary wave generation method is proposed while this setup has the potential to modify into pulsed EPR spectrometer to generate shaped pulses to manipulate electron spins state.

\section{Materials and methods}

In EPR spectroscopy, various frequency ranges are used. Among the frequency ranges used, $\mathrm{X}$ band is one of the common frequency ranges used in EPR. This frequency range has a variety of component choices because the technology for the commercial components is matured. Designing the EPR spectrometer in this frequency range reduces difficulties in designing and procuring the microwave components. For the microwave source, an FPGA is programmed into an AWG. Compared to a conventional AWG, the FPGA is more complex in designing the firmware, which is not as user friendly as the AWG. Apart from the FPGA, the resonator is also an important component in an EPR spectrometer. The resonator is used to induce a strong microwave magnetic field for B1. Depending on the resonator design, the EPR signal strength will be varied. Thus, the design and fabrication of the resonator in $\mathrm{X}$ band is part of this study.

As such, this study will start with designing and fabrication of the resonator, followed by spectrometer design which includes FPGA programming. In the resonator design, a simulation was done before the fabrication to estimate the specification of the resonator. A loop gap resonator (3 loop 2 gap) was designed because the loop gap resonator has moderate Q-factor, large filling factor, and high field homogeneity [15]. The resonance frequency of the resonator can be calculated using Equations (1):

$$
\omega_{0}=\frac{1}{2 \pi \sqrt{L C}}, \frac{1}{L}=\frac{1}{L_{1}}+\frac{1}{L_{2}}, L_{x}=\frac{n \mu_{0} \pi r_{x}^{2}}{Z}, C=\frac{\epsilon_{r} \epsilon_{0} W Z}{m t}
$$

In this equation, $\omega_{0}$ is the expected resonance frequency, $L$ is the total inductance, $L_{x}$ is the inductance of loop (where $\mathrm{x}=1,2,3$ ), $C$ is total capacitance, $n$ is the number of the identical loop, $\mu_{0}$ is the permeability of free space, $r_{x}$ is the radius of the loop, $Z$ is the height of the loop gap resonator, $\epsilon_{r}$ is relative dielectric constant, $\epsilon_{0}$ is the permittivity of free space, $W$ is the length of gaps, $m$ is the number of gaps and $t$ is the distance of gap. However, this equation does not include the cavity and coupling with the feed line. The simulated resonance frequency deviated from the calculated resonance frequency. The resonator design was done and shown in Figure 1. The resonator is capacitively coupled to the coaxial cable and the depth of the coaxial cable can change before the experiment to adjust the coupling to critical coupling. After the finite-difference time-domain method (FDTD) simulation result satisfied the requirements such as resonance frequency, Q-factor, and field homogeneity, the resonator was fabricated using the milling method. 

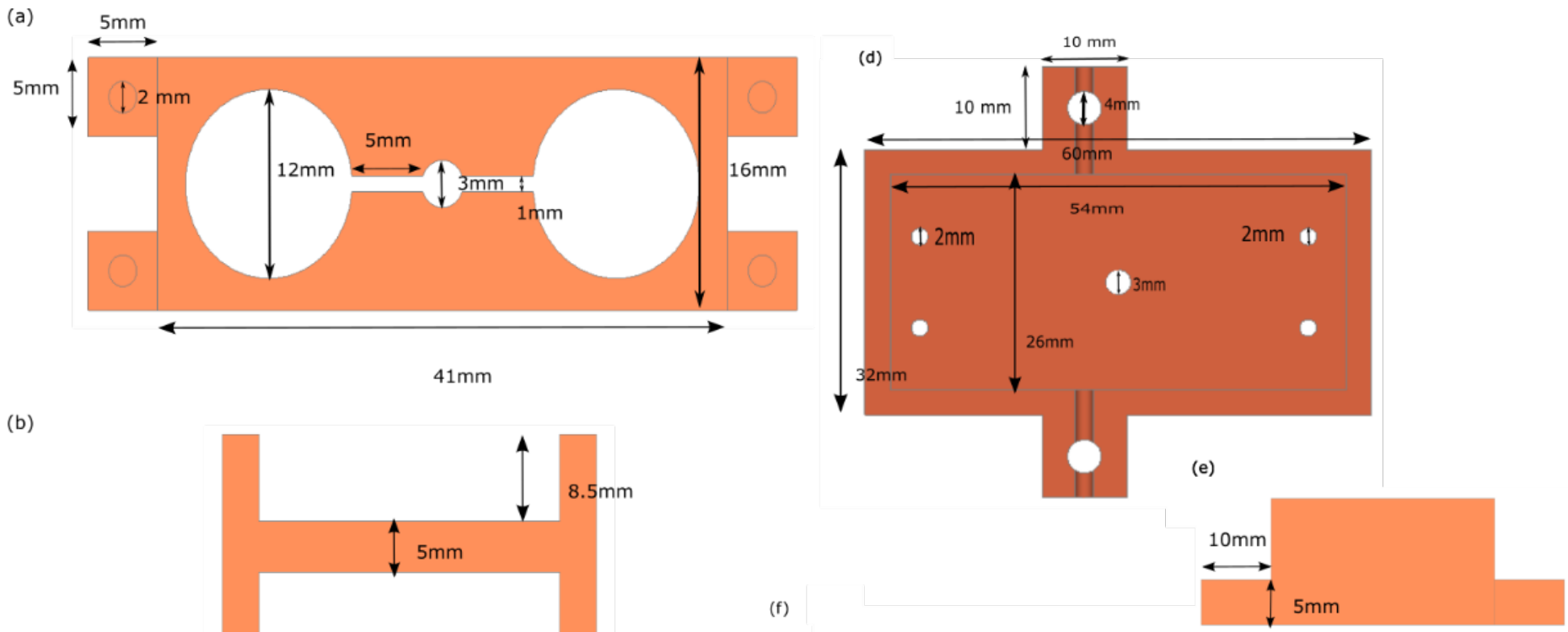

(b)

(c)

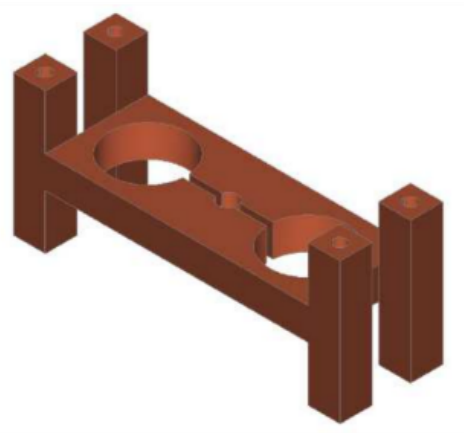

(f)

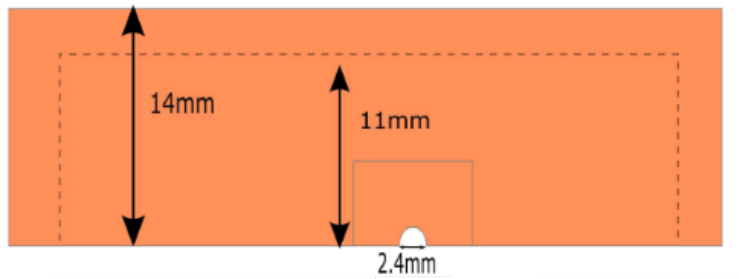

(g)

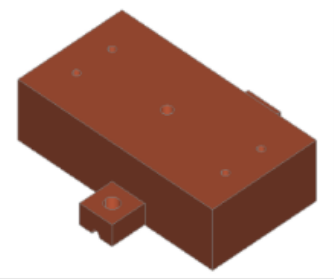

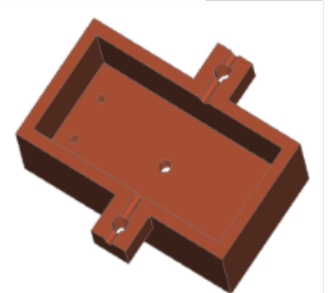

Figure 1. Design of the resonator and cavity. (a) Top view of the resonator. The resonator is symmetrical up and down, left and right with center at $3 \mathrm{~mm}$ loop. The holes with $2 \mathrm{~mm}$ diameter are thread holes for installation. (b) Front view of the resonator. (c) Overall view of the resonator. (d) Top view of the resonator cavity. The cavity design is symmetrical up and down with the center at $3 \mathrm{~mm}$ hole. The holes with $2 \mathrm{~mm}$ and $4 \mathrm{~mm}$ diameter are thread holes for installation. The cleavages at the top and bottom are the space to insert the feed line once the cavity is assembled. (e) Side view of the resonator cavity. (f) Front view of the resonator. The dotted line is the boundary of the empty cavity behind copper shielding. (g) Overall view of the cavity, two identical designs assemble form a complete cavity.

The spectrometer design starts with programming the FPGA. The FPGA used was Xilinx Kintex-7 KC705 with daughter card FMC 150. This FPGA was programmed to generate 0 to $122.5 \mathrm{MHz}$ of signal based on arbitrary wave generation method, which the $122.5 \mathrm{MHz}$ is the Nyquist frequency. The spectrometer design is shown in Figure 2. The IF signal generated by the FPGA undergo heterodyning and up converted to $X$ band RF signal. The RF signal is directed into the resonator and going out to the mixer for down conversion into the IF signal. The isolator in the spectrometer is to isolate the power reflected from the resonator to the components and damage or reflect from the mixer back to the resonator causing noise in the EPR signal. The effective bandwidth of this spectrometer is carefully designed to prevent the excitation of electron spin from the lower side band. The IF signal is generated by 16 bit DAC and sampled by 14 bit ADC of the FPGA. The IF signal is from 50 to $70 \mathrm{MHz}$, in a single frequency sweep, the spectrometer able to sweep from 3.8 to $10.07 \mathrm{GHz}$ due to the LO frequency is adjustable. However, there will be overlapping between the lower side band with upper side band. To prevent any overlapping of the lower and upper side band, the frequency range in a single frequency sweep was not exceed by $60 \mathrm{MHz}$ frequency. By using this frequency range and lower bound of $50 \mathrm{MHz}$ of IF signal, a $60 \mathrm{MHz}$ frequency gap is present between the upper limit frequency of the lower side band and the lower limit of the upper side band. By referring to Figure 3 (c), the $60 \mathrm{MHz}$ frequency gap is enough for the lower side band not to excite the electron spin due to the induced magnetic field at the sample area is weak. In other words, the lower side band away from the resonant frequency of the resonator increases the reflected power from the input of the resonator, which filters out the lower side band while allows only 
the upper side band signal to pass through and excites the electron spins. Before the start of the experiment, the spectrometer was loop back directly without using the resonator to check the signal purity.

(6)

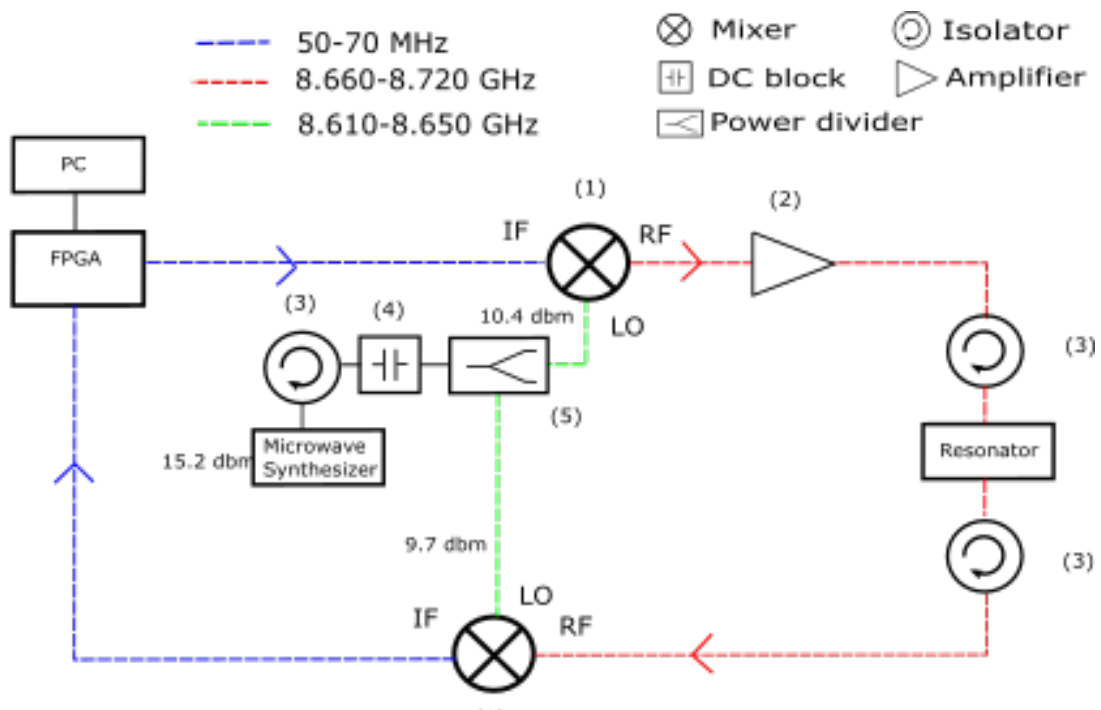

(1)

Figure 2. The setup for FPGA based EPR spectrometer. (1) Mini-circuit frequency mixer TB-MCA1-12GL+ where intermediate frequency (IF) is the input signal from FPGA, local oscillator (LO) is the input signal from microwave synthesizer and RF is the output signal. (2) Qorvo LNA QPA2609. (3) UIY isolator UIYCI1220A. (4) Mini-circuit DC block BLK-18-S+. (5) Mini-circuit power splitter TB-811+. The FPGA that is used are Xilinx FPGA Kintex-7 KC705 and daughter card FMC 150 with 16 bit DAC and 14 bit ADC. Microwave synthesizer is National Intrument FSL-0010.

In the continuous wave EPR experiment, the frequency sweep in the external magnetic field in on resonance condition and compared to the off resonance condition. If any EPR occurs, absorption will be observed, the absorption will be reflected in the lower amplitude of the continuous wave detected in the resonance condition compared to the off resonance condition. The magnetic field for resonance condition is estimated using Equation 2:

$$
h \omega=\mathrm{g} \mu_{B} B
$$

where $\mathrm{h}$ is the Plank's constant, $\mathrm{g}$ is the $\mathrm{g}$-factor of the sample (for this experiment, it is DPPH), $\mu_{B}$ is the Bohr magneton, $B$ is the external magnetic field strength, $\omega$ is the electron spins resonant frequency, which is the same as the resonant frequency of the resonator.

\section{Results and Discussion}

This part discusses the result of testing on resonator fabricated. Next, the resonator specifications such as resonant frequency and Q-factor are determined. After the resonator was shown it was working properly, the performances of the FPGA based spectrometer are discussed. Finally, the result of continuous wave EPR experiment by using the FPGA based spectrometer is presented.

\section{Performance of the Fabricated Resonator}

The FDTD simulation and experimental results of the resonator in Figure 3 shows that there are 2 peaks detected for both loaded and unloaded condition. For simulated unloaded conditions, the first peak at $8.350 \mathrm{GHz}$ is unwanted mode while $8.860 \mathrm{GHz}$ is the fundamental mode which is the resonance 
frequency of the resonator. To verify that $8.860 \mathrm{GHz}$ is the fundamental mode, the induced magnetic field is simulated. The FDTD simulation shows that the magnetic field is focused in the inner loop which is the sample area. This induced magnetic field distribution is the fundamental mode of the resonator [16].

(a)

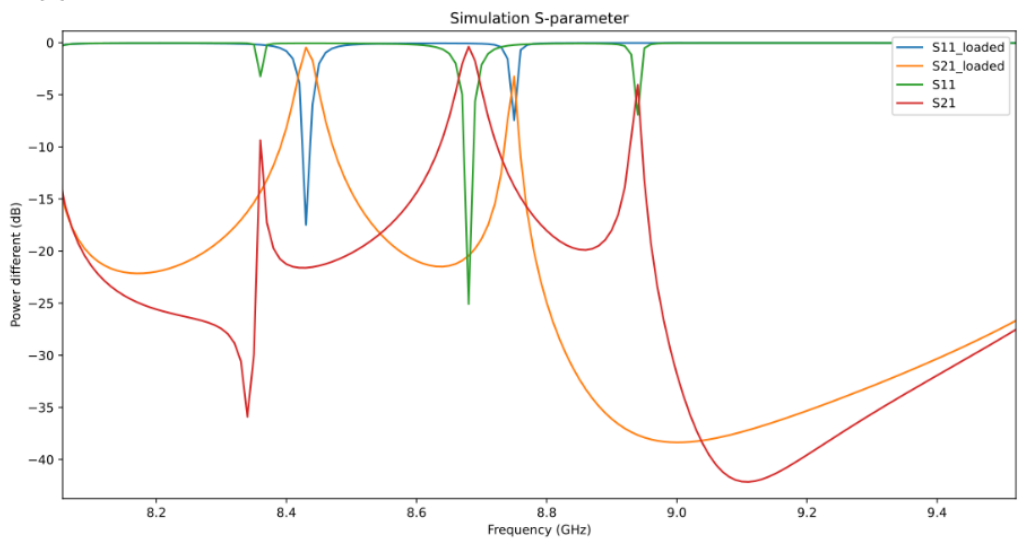

(b)

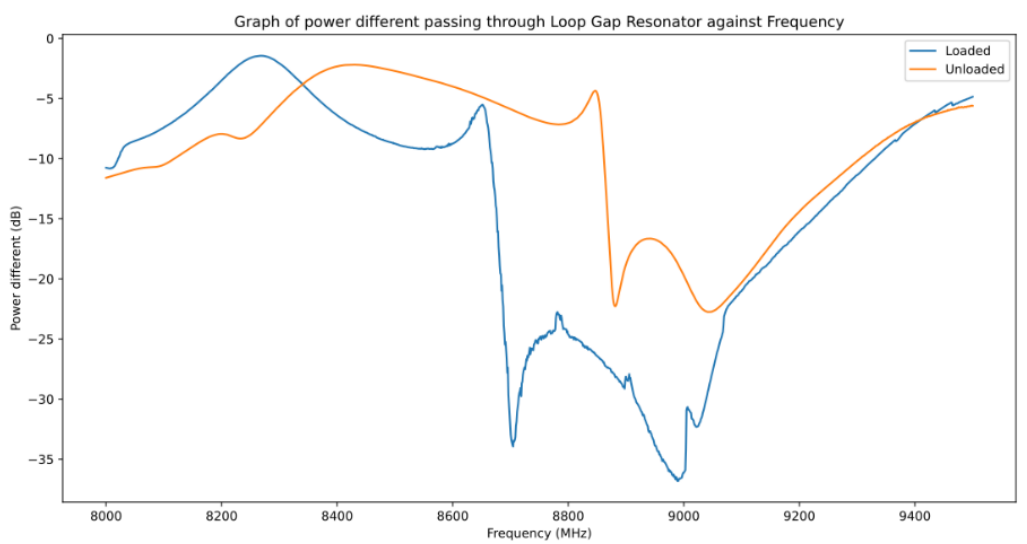

Figure 3. Simulation and experimental results of the resonator. (a) Simulation of s-parameter after applied AR-filter. (b) Experimental results of transmission loss passing through the resonator.

To save the simulation time, an autoregressive (AR) filter is applied as in Figure 3 (a). The AR filter is working by predicting the current simulation output with previous output and current input, this feature is commonly applied in electromagnetic simulation software. From Figure 3 (a), the s-parameter graph shows that the resonant frequency of the resonator decreases after loaded. This is because the sample used is DPPH in powder form. When the resonator is loaded, the glass sample holder is inserted into the inner loop of the resonator. Glass is a dielectric material and it increases the effective dielectric constant in the sample area, which decreases the resonant frequency. From experimental transmission loss of the resonator in Figure 3 (b), 2 peaks are detected for both loaded and unloaded conditions. This experimental result is overall agreed with the simulation result. The simulated and actual resonant frequencies and Q-factors are sort in Table 1 along with the calculated deviation between simulation and experiment. 
Table 1. The resonant frequency and Q-factor of the resonator in simulation and experiment. The deviation between experiment and simulation is calculated.

\begin{tabular}{lccc}
\hline & Simulation & Experiment & Discrepancy \\
\hline Unloaded Resonance Frequency & $8.860 \mathrm{GHz}$ & $8.852 \mathrm{GHz}$ & $8.0 \mathrm{MHz}$ \\
Loaded Resonance Frequency & $8.720 \mathrm{GHz}$ & $8.668 \mathrm{GHz}$ & $52.0 \mathrm{MHz}$ \\
Unloaded Q-factor & 812.7 & 646.0 & 166.7 \\
Loaded Q-factor & 794.8 & 615.8 & 179.0 \\
\hline
\end{tabular}

The simulated and actual specifications of the resonator do not deviate much from each other. The deviation of resonant frequencies and Q-factors is due to fabrication errors and additional parasitic capacitance induced by gaps between the parts of the resonator. For the loaded resonant frequency, the actual frequency deviated by $52 \mathrm{MHz}$ which is higher than the deviation of unloaded frequency. This is because the dielectric constant of the glass depends type of glass e.g. doping, fabrication method, etc. [17]. As such, the dielectric constant used in this simulation might be lower than the actual value. To determine the Q-factor, there are 2 methods. The first method is a continuous wave sweep method, this method is done by recording the transmission loss when continuous wave with different frequencies passing through the resonator. The second method is the ring down method, this method is using a pulse far from the resonance frequency to cause a ring down from the resonator. By analyzing the ring down, $\mathrm{Q}$-factor is determined from the ring down signal. This method will determine the effective Q-factor which is the combination of all resonance modes available in the resonator. Since the resonator is a lumped mode microwave structure, it has 2 resonance modes [16]. Thus, the ring down method might not be accurate, and continuous wave sweep method is chosen. The actual Q-factor lower than the simulated version might be due to the insulating oxide layer on the resonator. The oxide layer increases the resistance for surface current, which increases the losses. The gaps in between the assembly of the resonator also provide an additional channel of energy relaxation which decreases the Q-factor.

\section{FPGA based EPR spectrometer}

To ensure the EPR signal detected is by the designed frequency, the spectrometer was loop back directly at the resonant frequency, $8.869 \mathrm{GHz}$ without the resonator to check the signal purity as shown in Figure 4. In Figure 4(a), the designed frequency of the IF signal is $59 \mathrm{MHz}$ which is the dominant signal. A side band $\mathrm{f} 2$ with frequency $68 \mathrm{MHz}$ is detected and it is originated from FPGA. The harmonic of the dominant signal is also detected. After the IF signal passing through the mixers for up and down conversion, all the frequencies in the IF signal generated undergo 2 stages heterodyning. A total of 22 peaks are detected in Figures 4 (b) and (c) which originated from $f_{1}, f_{2}$, and $2 f_{1}$. Theoretically, there will be more than 22 peaks detected. The detected peaks are less than the theoretical value is because of 2 possibilities. The first possibility is the signal after first stage heterodyning become very weak and after the second stage heterodyning, the signal generated becoming too weak to be detected. The second possibility is the interference of multiple sources with the same frequency but different phases. Each peak may have multiple sources after heterodyning, those sources have different phases and undergo destructive interference and vanish or become too weak to be detected by ADC of FPGA. The calculated Spurious-Free Dynamic Range (SFDR) of the FPGA based spectrometer is $-13.3 \mathrm{dBc}$. 
(a)
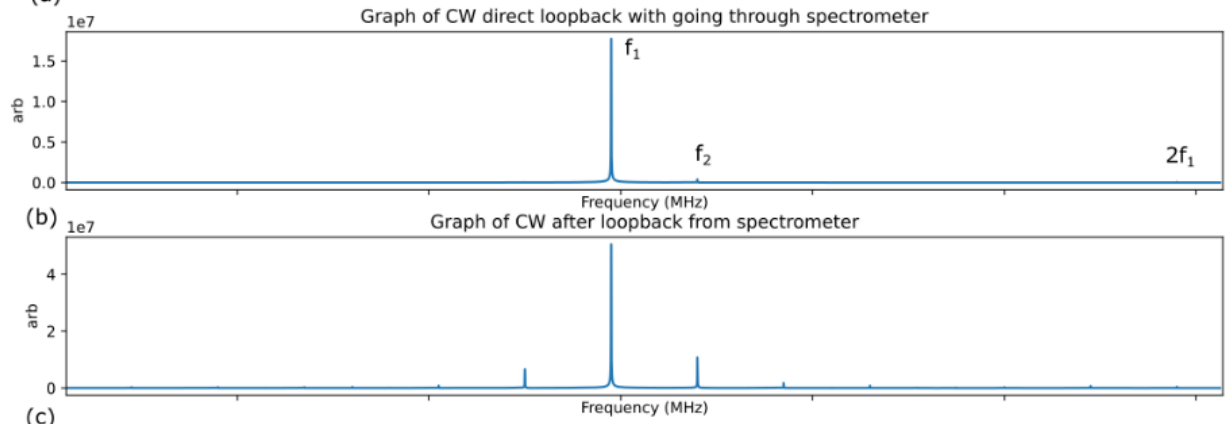

(c)

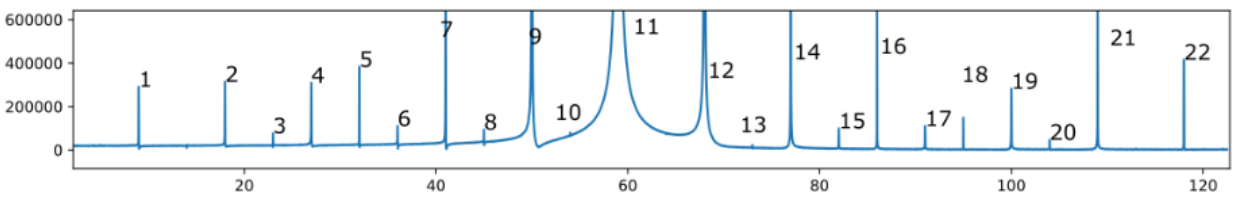

Figure 4. Signal generated by the FPGA based spectrometer. (a) Signal of CW direct loop-back from FPGA after Fast Fourier Transform. (b) Signal of CW loop-back from spectrometer without resonator after Fast Fourier Transform. (c) Display of all peaks detected in (b).

The EPR signal was detected by using the spectrometer design as shown in Figure 5. From the graph, the EPR signal is maximum at $311.4 \mathrm{mT}, 8.688 \mathrm{GHz}$. The EPR signal strength is increased from $311.2 \mathrm{mT}$ and achieves a maximum at $311.4 \mathrm{mT}$ then decreases. To verify the signal is the EPR signal, a frequency sweep at off resonance condition with the external magnetic field of $258.7 \mathrm{mT}$. From the spectrum, the EPR signal is vanished at off resonance condition, which shows that the EPR signal was detected at off resonance condition. The average signal to noise ratio (SNR) is $18 \pm 8$ under averaging of 4 and can improve by an increased number of averaging. The g-factor calculated by using Equation 2 is $1.9945 \pm 0.0012$, which deviated by $0.42 \%$ to the standard value of 2.003 and $0.23 \%$ to Chaudhuri [18]. The g-factor verified that this EPR signal is from DPPH but not from other sources. From the results above, the spectrometer design and the resonator are working well.

Figure 5. EPR signal from DPPH using FPGA based EPR spectrometer at different external magnetic fields. 


\section{Conclusion}

EPR has wide fields of application and its worth to study. The commercial products are unable to fit the requirements of some fields such as quantum computing. Thus, a FPGA based EPR spectrometer with a resonator is designed to carry out a continuous wave EPR experiment with DPPH as sample. The FPGA is used to replace the AWG to generate the driven magnetic field. This is because FPGA has low cost, low profile size and high flexibility. The frequency generated by the FPGA is up converted into $X$ band signal by heterodyning to excite the electron spins. Besides this, the FPGA detects and records the down converted signal that exited from the resonator. The resonator is designed, simulated and fabricated by using milling. The resonator fabricated has the loaded resonant frequency of $8.668 \mathrm{GHz}$ and Q-factor of 615.8 while the unloaded resonance frequency is $8.852 \mathrm{GHz}$ with a Q-factor of 646 . The design frequency of the FPGA based EPR spectrometer is the dominant frequency which is the frequency to excite electron spins with SFDR of $-13.3 \mathrm{dBc}$. EPR signal is detected and maximum at 311.4 $\mathrm{mT}, 8.688 \mathrm{GHz}$. The g-factor calculated is $1.9945 \pm 0.0012$ which is very near to the standard value. Therefore, the FPGA based spectrometer and the resonator designed are working for continuous experiment. For the future work, the spectrometer will extend to pulsed spectrometer by installing suitable pulsed amplifier and attenuator. Various pulsed EPR experiments and qubit operations can be done to determine the spin dynamic of the electron spins.

\section{Data availability}

The data can be requested from the first author by email: siang72111@gmail.com or yungszen@utm.my

\section{Conflicts of Interest}

The author(s) declare(s) that there is no conflict of interest regarding the publication of this paper.

\section{Acknowledgment}

This work was financially supported by Fundamental Research Grant Scheme (FRGS), Malaysia (FRGS/1/2018/STG02/UTM/02/15 and FRGS/1/2021/STG07/UTM/03/1) by the Ministry of Higher Education (MOHE) and UTM (R.J130000.7854.5F027, Q.J130000.2626.11J23, QJ130000.3554.06G13 and R.J130000.7854.5F427). A faithfully thanks to Dr. Ganesan A/L Krishnan, Dr. Muhammad Safwan Abd Aziz and Dr Ezza Syuhada Sazali for providing precious advice and research grant support to this study. Without anyone of them, this study was not able to complete.

\section{References}

[1] F. Shi, Q. Zhang, P.Wang, et al., "Single-protein spin resonance spectroscopy under ambient conditions," Science, vol. 347, no. 6226, pp. 1135\{1138, 2015.

[2] L. Mainali, J. W. Sidabras, T. G. Camenisch, et al. "Spin-label W-band EPR with seven-loop-six-gap resonator: application to lens membranes derived from eyes of a single donor," Applied magnetic resonance, vol. 45, no. 12 , pp. $1343\{1358,2014$

[3] V. R. Horowitz, B. J. Aleman, D. J. Christle, A. N. Cleland, and D. D. Awschalom, "Electron spin resonance of nitrogen-vacancy centers in optically trapped nanodiamonds," Proceedingsof the National Academy of Sciences, vol. 109, no. 34, pp. 13 493-13 497, 2012.

[4] D. M. Zajac, A. J. Sigillito, M. Russ, et al, "Resonantly driven CNOT gate for electron spins," Science, vol. 359, no. 6374 , pp. 439-442, 2018. 7.

[5] E. Vahapoglu, J. Slack-Smith, R. Leon, et al., "Coherent control of electron spin qubits in silicon using a global field," arXiv preprint arXiv:2107.14622, 2021.

[6] H. Toida, Y. Matsuzaki, K. Kakuyanagi,et al, "Electron paramagnetic resonance spectroscopy using a single artificial atom," Communications Physics, vol. 2, no. 1, pp. 1-7, 2019.

[7] A. Bienfait, J. J. Pla, Y. Kubo, et al, "Reaching the quantum limit of sensitivity in electron spin resonance," Nature Nanotechnology, vol. 11, p. 253, 2015. [Online]. Available: http://dx.doi.org/10.1038/nnano.2015.282.

[8] P. P. Borbat, R. H. Crepeau, and J. H. Freed, "Multifrequency two-dimensional fourier transform esr: an x/kuband spectrometer," Journal of Magnetic Resonance, vol. 127, no. 2, pp. 155-167, 1997

[9] A. Formanuik, A.-M. Ariciu, F. Ortu, et al, "Actinide covalency measured by pulsed electron paramagnetic 
resonance spectroscopy," Nature chemistry, vol. 9, no. 6, p. 578, 2017

[10] A. Band, M. P. Donohue, B. Epel, S. Madhu, and V. A. Szalai, "Integration of a versatile bridge concept in a 34 ghz pulsed/cw EPR spectrometer," Journal of Magnetic Resonance, vol. 288, pp. 28-36, 2018.

[11] J. J. Morton, A. M. Tyryshkin, A. Ardavan, et al, "High fidelity single qubit operations using pulsed electron paramagnetic resonance," Physical review letters, vol. 95, no. 20, p. 200501, 2005.

[12] S. Probst, V. Ranjan, Q. Ansel, et al., "Shaped pulses for transient compensation in quantum-limited electron spin resonance spectroscopy," Journal of Magnetic Resonance, vol. 303, pp. 42\{47, 2019

[13] Y. S. Yap, M. Negoro, M. Kuno, et al, "Low power, fast and broadband ESR quantum control using a stripline resonator," arXiv preprint arXiv:1911.04314, 2019

[14] H. Sato-Akaba and M. Tseytlin, "Development of an I-band rapid scan epr digital console," Journal of Magnetic Resonance, vol. 304, pp. 42-52, 2019.

[15] G. A. Rinard and G. R. Eaton, "Loop-gap resonators," in Biomedical EPR, Part B: Methodology, Instrumentation, and Dynamics. Springer, 2005, pp. 19-52.

[16] R. L. Wood, W. Froncisz, and J. S. Hyde, "The loop-gap resonator. II. Controlled return flux three-loop, two-gap microwave resonators for ENDOR and ESR spectroscopy," Journal of Magnetic Resonance (1969), vol. 58, no. 2, pp. 243-253, 1984.

[17] J. Chen, "La doping effect on the dielectric property of barium strontium titanate glass-ceramics," Journal of Materials Science \& Technology, vol. 30, pp. 295-298, 2014.

[18] U. Chaudhuri and R. Mahendiran, "Detection of L-band electron paramagnetic resonance in the DPPH molecule using impedance measurements," RSC Advances, vol. 10, no. 29, pp.17 311-17316, 2020. 